\title{
Granulomatous Pachymeningitis Around the Sella Turcica (Tolosa-Hunt Syndrome) Involving the Hypophysis
}

\author{
-Case Report-
}

\author{
Daisuke KITA, Osamu TACHIBANA, Yukihiro NAGAI*, \\ Hiroki SANO, and Junkoh YAMASHITA \\ Department of Neurosurgery, Graduate School of Medical Science, Kanazawa University, \\ Kanazawa, Ishikawa; * Department of Internal Medicine, Kanazawa \\ Municipal Hospital, Kanazawa, Ishikawa
}

\begin{abstract}
A 50-year-old female presented with right painful abducens nerve palsy persisting for 4 months and mild panhypopituitarism with diabetes insipidus for 6 months. $T_{1}$-weighted magnetic resonance (MR) imaging of the sellar region showed a homogeneously enhanced mass lesion in the right cavernous sinus which seemed to extend from the swollen pituitary gland. $T_{2}$-weighted MR imaging clearly showed the mass in the right cavernous sinus and the thickened dura mater of the sellar floor as hypointense, and the enlarged pituitary gland as isointense. Biopsy of the thickened dura mater and swollen pituitary gland was performed via the transsphenoidal approach. Histological examination revealed inflammation and collagen fiber formation in these regions. The diagnosis was secondary panhypophysitis resulting from granulomatous pachymeningitis involving the cavernous sinus (Tolosa-Hunt syndrome). Corticosteroid therapy was begun after the biopsy. Her periorbital pain and diplopia were relieved, but diabetes insipidus persisted. Follow-up MR imaging showed a decrease in the volumes of the pituitary gland and the mass in the cavernous sinus.
\end{abstract}

Key words: Tolosa-Hunt syndrome, pachymeningitis, panhypophysitis, diabetes insipidus

\section{Introduction}

Tolosa-Hunt syndrome manifests as an episodic orbital pain associated with paralysis of one or more of the third, fourth, or sixth cranial nerves. ${ }^{6)}$ TolosaHunt syndrome represents some of the symptoms of idiopathic cranial pachymeningitis involving the cavernous sinuses and surrounding cranial nerves. ${ }^{9)}$ The pituitary gland is rarely involved in this syndrome. Here we report a case of secondary panhypophysitis associated with Tolosa-Hunt syndrome.

\section{Case Report}

A 50-year-old woman complained of right perior- bital pain and diplopia. Two months later, she began to complain of intolerable headaches with general malaise, as well as polydipsia and polyuria. Accordingly, she was admitted to a local hospital. Physical examination on admission showed obesity (height $148 \mathrm{~cm}$, weight $74.0 \mathrm{~kg}$, body mass index 33.8). There was pain and tenderness over the right forehead but the eye ball was neither tender nor tense. Neurological examination revealed right abducens nerve palsy. No meningeal irritation signs were detected. Blood and cerebrospinal fluid (CSF) examinations found no signs of systemic or neuronal inflammation. Anti-thyroid and anti-nuclear antibodies were not found in the serum. Anti-pituitary gland antibody was also not found in both the serum and CSF.

Received February 6, 2006; Accepted October 24, 2006

Authors' present addresses: D. Kita, M.D., Pathology Group, International Agency for Research on Cancer (IARC), World Health Organization (WHO), Lyon, France; O. Tachibana, M.D., Department of Neurosurgery, Kanazawa Medical University, Kahoku-gun, Ishikawa, Japan; Y. Nagai, M.D., Nagai Medical Clinic, Kanazawa, Ishikawa, Japan; J. Yamashita, M.D., President, Osaka Saiseikai Izuo Hospital, Osaka, Japan. 
Table 1 Water deprivation test with exogenous antidiuretic hormone administration test before prednisolone pulse therapy

\begin{tabular}{lrrrrrrrr}
\hline & 1 & 2 & 3 & 4 & 5 & 6 & 7 & $8 \mathrm{hrs}$ \\
\hline Urine volume (ml/hr) & 350 & 200 & 150 & 290 & 150 & 100 & 17 & 25 \\
Urine osmotic pressure (mOsm/l) & 61 & 65 & 74 & 74 & 79 & 95 & 117 & 305 \\
\hline
\end{tabular}

High urine volume with low urine osmotic pressure continued for 5 hours, when 5 IU of soluble deamino arginine vasopressin was administered subcutaneously.

Table 2 Simultaneous three-hormone loading test before corticosteroid therapy

\begin{tabular}{|c|c|c|c|c|c|c|}
\hline & Normal range & 0 & 30 & 60 & 90 & $120 \mathrm{~min}$ \\
\hline $\mathrm{TSH}(\mu \mathrm{IU} / \mathrm{ml})$ & $0.27-4.65$ & 0.62 & 4.29 & 3.27 & 2.30 & 1.38 \\
\hline $\mathrm{FSH}(\mathrm{mIU} / \mathrm{ml})$ & $2.7-22.9$ & 2.2 & 10.8 & 13.0 & 14.4 & 14.7 \\
\hline $\mathrm{GH}(\mathrm{ng} / \mathrm{ml})$ & $<3.0$ & 0.47 & 0.65 & 2.26 & 1.85 & 0.78 \\
\hline ACTH $(\mathrm{pg} / \mathrm{ml})$ & $<46.0$ & 5.7 & 9.1 & 19.3 & 21.9 & 22.1 \\
\hline
\end{tabular}

$500 \mu \mathrm{g}$ of thyrotropin-releasing hormone, $100 \mu \mathrm{g}$ of luteinizing hormone (LH)-releasing hormone, and 5 IU of regular insulin were simultaneously administered intravenously. ACTH: adrenocorticotrophic hormone, FSH: folliclestimulating hormone, GH: growth hormone, PRL: prolactin, TSH: thyroid-stimulation hormone.

Pituitary function was evaluated with the water deprivation test and simultaneous three-hormone administration test. The water deprivation test followed by exogenous antidiuretic hormone administration indicated central type diabetes insipidus (Table 1). The simultaneous thyrotropin-releasing hormone, luteinizing hormone-releasing hormone, and insulin loading test showed hyposecretion of thyroid-stimulating hormone, follicle-stimulating hormone, luteinizing hormone, growth hormone, adrenocorticotrophic hormone, and cortisol, as well as hypersecretion of prolactin, indicating mild malfunction of the anterior pituitary gland (Table 2).

Sagittal $\mathrm{T}_{1}$-weighted magnetic resonance (MR) imaging showed the swollen pituitary gland without a high signal intensity area in the posterior pituitary gland, consistent with central type diabetes insipidus $^{2)}$ (Fig. 1A). Coronal MR imaging of the sellar region suggested pituitary macroadenoma with cavernous sinus infiltration (Fig. 1B, C). On the other hand, $\mathrm{T}_{2}$-weighted $\mathrm{MR}$ imaging showed a hypointense mass in the right cavernous sinus, which was clearly discriminated from the enlarged pituitary gland (Fig. 1E). $\mathrm{T}_{2}$-weighted $\mathrm{MR}$ imaging showed the dura mater at the sellar region as hypointense and thickened (Fig. 1D, E). Computed tomography did not show any high density area or bone erosion around the sellar region (not shown).

The most probable diagnosis was granulomatous inflammation in the right cavernous sinus wall affecting both the adjacent dura mater and pituitary gland. A biopsy was carried out via the transsphenoidal approach to exclude other paracavernous syndromes, such as benign tumor or infectious

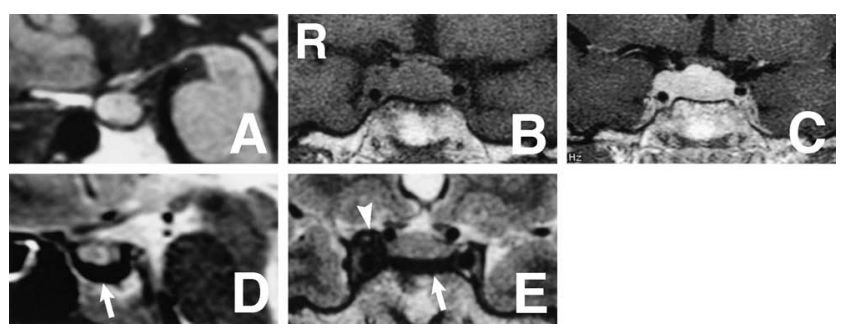

Fig. 1 Magnetic resonance (MR) images taken 4 months after the onset. Sagittal $T_{1}$-weighted MR image (A) showing enlargement of the pituitary gland, and absence of the normal hyperintense area in the posterior part of the pituitary gland, indicating absence of antidiuretic hormone. Coronal $\mathrm{T}_{1}$-weighted MR images (B) and with gadolinium (C) showing the pituitary gland and the right cavernous sinus as isointense (B), with homogeneous enhancement (C). Sagittal (D) and coronal (E) $T_{2}$-weighted $M R$ images clearly showing the dura mater at the sellar floor (arrow) and the granuloma in the right cavernous sinus (arrowhead) as hypointense indicating inflammation and fibrous change. 


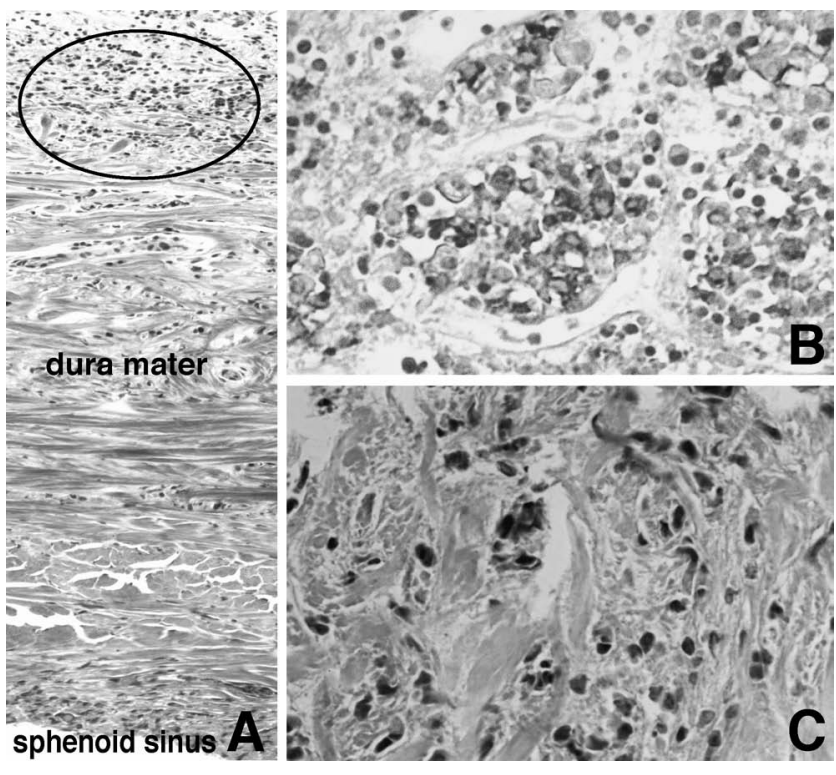

Fig. 2 Photomicrographs of the biopsy specimens. (A) The dura mater was thickened with abundant collagen bundles (approximately $3 \mathrm{~mm}$ in diameter). Inflammatory cells had infiltrated into the dura mater and the adjacent anterior pituitary gland (circled). Hematoxylin and eosin (HE) stain, $\times 40$. (B) Immunohistochemical prolactin staining showing preservation of the acinus formation in the anterior pituitary gland, despite the inflammatory process. $\times 200$. (C) Infiltration of inflammatory cells and increased collagen fibers in the posterior pituitary gland, indicating chronic inflammation. HE stain, $\times 400$.

inflammatory masses. Intraoperatively, we observed marked thickening of the dura mater (approximately $3 \mathrm{~mm}$ ). The anterior lobe of the pituitary gland was soft and yellowish, whereas the posterior lobe was elastic and hard. Specimens from the granulomatous lesion in the cavernous sinus were not obtained to avoid bleeding. Histological examination of the excised dura mater showed abundant collagen bundles with hyalinization which had markedly thickened the dura mater. Infiltration of inflammatory cells was also observed in the dura mater, mostly on the side of the pituitary gland (Fig. 2A). Immunohistochemical studies revealed that these inflammatory cells were mainly CD8-positive lymphocytes, i.e. cytotoxic $\mathrm{T}$ cells (not shown). The inflammatory lymphocytes were also found in the anterior pituitary gland, whereas the acinus formations were almost completely preserved as clearly shown by immunohistochemical staining with antiprolactin antibody (Fig. 2B). Tissues in the posterior pituitary gland were displaced by increased collagen

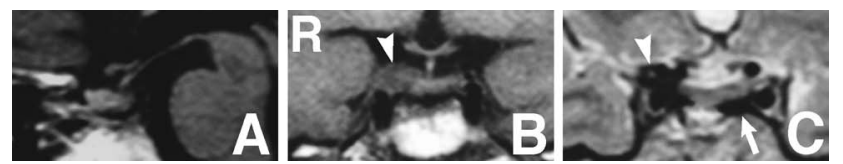

Fig. 3 Magnetic resonance (MR) images taken 6 months after corticosteroid therapy. Sagittal (A) and coronal (B) $T_{1}$-weighted $M R$ images and coronal (C) $\mathrm{T}_{2}$-weighted $\mathrm{MR}$ image showing the pituitary gland had shrunk, although the normal hyperintense area in the posterior part of the pituitary gland remained undetectable $(A)$. The granuloma in the right cavernous sinus (arrowhead) and the thickening of the dura mater (arrow) also persisted (B, C).

fibers together with inflammatory cells, similar to the dura mater (Fig. 2C).

After the occurrence of non-specific inflammatory processes around the sellar region was confirmed, she began receiving regular corticosteroid therapy (orally prednisolone $20 \mathrm{mg} /$ day). Her painful diplopia and general malaise were relieved, and she continued to receive intranasal desmopressin for diabetes insipidus. Six months later, follow-up MR imaging showed a decrease in the volumes of the pituitary gland and the granuloma in the cavernous sinus (Fig. 3).

\section{Discussion}

Thickened wall of the cavernous sinus accompanied by granulomatous soft tissue mass is often observed in the radiological study of patients with TolosaHunt syndrome. ${ }^{1)}$ Histological studies of the abnormal lesions reveal non-specific inflammation in meninges, compatible with pachymeningitis. ${ }^{3,4,8,9)}$ Tolosa-Hunt syndrome is considered to represent some of the symptoms of idiopathic cranial pachymeningitis involving the cavernous sinuses and surrounding cranial nerves. ${ }^{9)}$

The differential diagnosis of Tolosa-Hunt syndrome should include other paracavernous diseases, such as pituitary macroadenoma with cavernous sinus infiltration, paracavernous meningioma, Meckel's cave neurinoma, suprasellar epidermoid tumor, leptomeningeal metastases, granulomatous pachymeningitis sequelae, and aneurysm causing compression of the cavernous sinus. ${ }^{1)}$ In our case, $\mathrm{T}_{1}$-weighted MR imaging of the sellar region indicated pituitary macroadenoma with cavernous sinus infiltration (Fig. 1A-C). We wish to emphasize the fact that $\mathrm{T}_{2}$-weighted $M R$ imaging was very useful for distinguishing the lesions located in the extrapituitary gland from those in the pituitary gland. The 
hypointense lesions on the $\mathrm{T}_{2}$-weighted $\mathrm{MR}$ images were related to the degree of fibrosis because of the absence of mobile protons due to the fibrotic background and/or high cellularity of the lesion.4) In our case, the hypertrophic dura mater was clearly shown as hypointense on the $\mathrm{T}_{2}$-weighted $\mathrm{MR}$ images (Fig. 1D, E), and was well correlated with the histological findings of increased collagen bundles (Fig. 2A).

Lymphocytic adenohypophysitis with granulomatous lesion should be considered in the differential diagnosis, which might involve the surrounding meningis and the posterior pituitary gland. ${ }^{10)}$ However, radiological study clearly showed that the granulomatous lesion was outside the pituitary gland. In addition, the histological study showed that the anterior pituitary gland was not so affected compared with the posterior lobe, which correlated well with the hormonal evaluation. These findings suggest that the inflammation may not have originated from the anterior pituitary gland. Lymphocytic infundibuloneurohypophysitis may also cause idiopathic diabetes insipidus, with lymphocytic infiltration of the pituitary stalk and destruction of the entire pituitary gland.7) The histological features of this disease are similar to those in our case, although we consider our case as a separate entity because of the patient's age, lack of correlation with pregnancy, and paracavernous spread of the inflammation.

Only three cases of diabetes insipidus in association with Tolosa-Hunt syndrome have been reported. ${ }^{3,5,8)}$ All patients presented with severe panhypopituitarism. Histological examinations of the pituitary gland showed marked increases of collagen fibers and inflammatory cells in the dura mater and the entire pituitary gland. Our case might represent an early stage of the inflammatory process in terms of the anterior pituitary gland, because both the function and structure of the anterior pituitary gland were not severely disturbed. Whether the inflammation originated from the cavernous sinus, the dura mater of the sellar floor, or the pituitary gland was not clear, but both the dura mater and the pituitary gland had identical histological features. ${ }^{8)}$ Such similarity of histological findings in the dura mater and the posterior pituitary gland strongly suggest the same chronic inflammatory process. In addition, the diabetes insipidus was followed by Tolosa-Hunt syndrome on the right in this patient.

The present rare case of secondary panhypophysitis originating from granulomatous pachymeningitis around the sella turcica indicated that inflammation in the right cavernous sinus and sellar floor spread mainly into the posterior pituitary gland, resulting in permanent diabetes insipidus and mild hypofunction of anterior pituitary gland.

\section{Acknowledgment}

We thank Ms. Imamura for her admirable preparation of the tissue samples, and Dr. Refaat Mohamed (Department of Neurosurgery, Cairo University, Cairo, Egypt) and Mr. Stephen Martin for their critical reading of this manuscript.

\section{References}

1) Cakirer S: MRI findings in the patients with the presumptive clinical diagnosis of Tolosa-Hunt syndrome. Eur Radiol 13: 17-28, 2003

2) Fujisawa I, Asato R, Okumura R, Nakano Y, Shibata T, Hamanaka D, Hashimoto T, Konishi J: Magnetic resonance imaging of neurohypophyseal germinomas. Cancer 68: 1009-1014, 1991

3) Hama S, Arita K, Kurisu K, Sumida M, Kurihara K: Parasellar chronic inflammatory disease presenting Tolosa-Hunt syndrome, hypopituitarism and diabetes insipidus: a case report. Endocr J 43: 503-510, 1996

4) Han MH, Chi JG, Kim MS, Chang KH, Kim KH, Yeon KM, Han MC: Fibrosing inflammatory pseudotumors involving the skull base: MR and CT manifestations with histopathologic comparison. AJNR Am J Neuroradiol 17: 515-521, 1996

5) Hida C, Yamamoto T, Endo K, Tanno Y, Saito T, Tsukamoto $\mathrm{T}$ : Inflammatory involvement of the hypophysis in Tolosa-Hunt syndrome. Intern Med 34: 1093-1096, 1995

6) Hunt WE: Tolosa-Hunt syndrome: one cause of painful ophthalmoplegia. J Neurosurg 44: 544-549, 1976

7) Imura H, Nakao K, Shimatsu A, Ogawa Y, Sando T, Fujisawa I, Yamabe $\mathrm{H}$ : Lymphocytic infundibuloneurohypophysitis as a cause of central diabetes insipidus. N Engl J Med 329: 683-689, 1993

8) Kitai R, Sato K, Kubota T, Kabuto M, Uno $H$, Kobayashi H: Hypertrophic cranial pachymeningitis involving the pituitary gland: a case report. Surg Neurol 48: 58-63, 1997

9) Miwa H, Koshimura I, Mizuno Y: Recurrent cranial neuropathy as a clinical presentation of idiopathic inflammation of the dura mater: a possible relationship to Tolosa-Hunt syndrome and cranial pachymeningitis. J Neurol Sci 154: 101-105, 1998

10) Yoshioka M, Yamakawa $N$, Saito $H$, Yoneda $M$, Nakayama T, Kuroki M, Tsuchida T, Sekiya M: Granulomatous hypophysitis with meningitis and hypopituitarism. Intern Med 31: 1147-1150, 1992

Address reprint requests to: Daisuke Kita, M.D., Department of Neurosurgery, Graduate School of Medical Science, Kanazawa University, 13-1 Takara-machi, Kanazawa 920-8641, Japan. e-mail: kitad@ns.m.kanazawa-u.ac.jp 